International Journal of Applied Mathematics

Volume 26 No. $3 \quad 2013,309-320$

ISSN: 1311-1728 (printed version); ISSN: 1314-8060 (on-line version)

doi: http://dx.doi.org/10.12732/ijam.v26i3.6

\title{
ON CORRECTORS FOR SPECTRAL PROBLEMS IN THE HOMOGENIZATION OF ROBIN BOUNDARY CONDITIONS WITH VERY LARGE PARAMETERS
}

\author{
Delfina Gómez ${ }^{1}$, Miguel Lobo ${ }^{1}$, Eugenia Pérez ${ }^{2}$, \\ Tatiana A. Shaposhnikova ${ }^{3}$ \\ ${ }^{1}$ Dpto. de Matemáticas, Estadística y Computación \\ Universidad de Cantabria \\ Santander - 39005, SPAIN \\ ${ }^{2}$ Dpto. de Matemática Aplicada y Ciencias de la Computación \\ Universidad de Cantabria \\ Santander - 39005, SPAIN \\ ${ }^{3}$ Department of Differential Equations \\ Moscow State University \\ Moscow, 119992, RUSSIA
}

\begin{abstract}
We obtain estimates for convergence rates of the eigenelements $\left(\lambda^{\varepsilon}, u^{\varepsilon}\right)$ for the Laplace operator in a domain $\Omega \subset \mathbb{R}^{3}$ periodically perforated along a plane $\gamma=\Omega \cap\left\{x_{1}=0\right\}$. The boundary conditions are of the Dirichlet type on $\partial \Omega$ and of the Robin type, involving a large parameter $O\left(\varepsilon^{-\kappa}\right)$, on the boundary of the cavities. The small parameter $\varepsilon$ denotes the period while the size of each cavity is $O\left(\varepsilon^{\alpha}\right)$. Here we consider the most significant case where $\alpha=\kappa=2$.
\end{abstract}

AMS Subject Classification: 35B27, 35B40, 35P05, 35P15

Key Words: boundary homogenization, porous media, spectral analysis, asymptotic analysis

\section{Introduction and Setting of the Problem}

Let $\Omega$ be a bounded domain in $\mathbb{R}^{3}$, with a smooth boundary $\partial \Omega$. Assume that

Received: April 29, 2013

(C) 2013 Academic Publications

$\S_{\text {Correspondence author }}$ 
$\gamma=\Omega \cap\left\{x_{1}=0\right\} \neq \emptyset$ is a domain on the plane $\left\{x_{1}=0\right\}$. We denote by $G_{0}$ the ball of radius 1 centered at the origin of coordinates. For a domain $B$, and $\delta>0$, we denote by $\delta B=\left\{x \mid \delta^{-1} x \in B\right\}$, and we set

$$
\widetilde{G}_{\varepsilon}=\bigcup_{z \in \mathbb{Z}^{\prime}}\left(a_{\varepsilon} G_{0}+\varepsilon z\right) \equiv \bigcup_{j \in \mathbb{Z}^{\prime}} G_{\varepsilon}^{j},
$$

where $\mathbb{Z}^{\prime}$ is the set of points of the form $z=\left(0, z_{2}, z_{3}\right)$ with integer components $z_{2}, z_{3} ; a_{\varepsilon}=C_{0} \varepsilon^{\alpha}, C_{0}$ is a fixed positive number, $\varepsilon>0$ is a parameter that we make converging towards zero, and $\alpha \geq 1$. We define

$$
G_{\varepsilon}=\bigcup_{j \in \Upsilon_{\varepsilon}} G_{\varepsilon}^{j}
$$

where $\Upsilon_{\varepsilon}=\left\{j \in \mathbb{Z}^{\prime}: G_{\varepsilon}^{j} \subset \widetilde{G}_{\varepsilon}, \bar{G}_{\varepsilon}^{j} \subset \Omega, \rho\left(\partial \Omega, \bar{G}_{\varepsilon}^{j}\right) \geq 2 \varepsilon\right\}$. The number of $G_{\varepsilon}^{j}$ with index $j \in \Upsilon_{\varepsilon}$ is $\quad\left|\Upsilon_{\varepsilon}\right|=O\left(\varepsilon^{-2}\right)$.

Let $\Omega_{\varepsilon}$ be $\Omega_{\varepsilon}=\Omega \backslash \bar{G}_{\varepsilon}, S_{\varepsilon}=\partial G_{\varepsilon}, \partial \Omega_{\varepsilon}=\partial \Omega \cup S_{\varepsilon}$. Let $H^{1}\left(\Omega_{\varepsilon}, \partial \Omega\right)$ be the space completion with respect to the norm of $H^{1}\left(\Omega_{\varepsilon}\right)$ of the set of functions $u \in \mathcal{C}^{\infty}\left(\bar{\Omega}_{\varepsilon}\right), u$ vanishing in a neighborhood of $\partial \Omega$.

Let us consider the eigenvalue problem

$$
\begin{cases}-\Delta u^{\varepsilon}=\lambda^{\varepsilon} u^{\varepsilon} & \text { in } \Omega_{\varepsilon} \\ u^{\varepsilon}=0 & \text { on } \partial \Omega \\ \partial_{\nu} u^{\varepsilon}+\varepsilon^{-\kappa} a u^{\varepsilon}=0 & \text { on } S_{\varepsilon}\end{cases}
$$

where $\partial_{\nu}$ denotes the derivative along the unit outward normal vector $\nu$ to $\partial \Omega_{\varepsilon}$ on $S_{\varepsilon}, a \equiv a(x)$ is a strictly positive continuously differentiable function in $\bar{\Omega}$ and $\kappa \in \mathbb{R}$. Throughout this paper, we set the value of the parameters $\alpha$ and $\kappa$ at $\kappa=\alpha=2$.

The variational formulation of (1) is: to find $\lambda^{\varepsilon}, u^{\varepsilon} \in H^{1}\left(\Omega_{\varepsilon}, \partial \Omega\right), u^{\varepsilon} \neq 0$, such that

$$
\int_{\Omega_{\varepsilon}} \nabla u^{\varepsilon} \nabla v d x+\varepsilon^{-\kappa} \int_{S_{\varepsilon}} a u^{\varepsilon} v d s=\lambda^{\varepsilon} \int_{\Omega_{\varepsilon}} u^{\varepsilon} v d x, \quad \forall v \in H^{1}\left(\Omega_{\varepsilon}, \partial \Omega\right) .
$$

For each fixed $\varepsilon>0$, problem (2) is a standard eigenvalue problem in the couple of spaces $H^{1}\left(\Omega_{\varepsilon}, \partial \Omega\right) \subset L^{2}\left(\Omega_{\varepsilon}\right)$, with a discrete spectrum. Let us consider $\left\{\lambda_{k}^{\varepsilon}\right\}_{k=1}^{\infty}$ the increasing sequence of eigenvalues, repeated according to their multiplicities, and let $\left\{u_{k}^{\varepsilon}\right\}_{k=1}^{\infty}$ denote the associated eigenfunctions which are assumed to form an orthonormal basis in $L^{2}\left(\Omega_{\varepsilon}\right)$. 
As stated in Gómez et al [1], for $\alpha=\kappa=2$, the homogenized spectral problem of (1) is:

$$
\begin{cases}-\Delta u=\lambda u & \text { in } \Omega^{-} \cup \Omega^{+}, \\ u=0 & \text { on } \partial \Omega, \\ {[u]=0,\left[\partial_{x_{1}} u\right]=4 \pi C_{0} h u} & \text { on } \gamma,\end{cases}
$$

where $h \equiv h(x)$ is the strictly positive continuously differentiable function defined by

$$
h(x)=\frac{a(x) C_{0}}{1+a(x) C_{0}}, \quad x \in \bar{\Omega} .
$$

Problem (3) has a discrete spectrum; let us consider $\left\{\lambda_{k}\right\}_{k=1}^{\infty}$ the increasing sequence of its eigenvalues repeated according to their multiplicities, and let $\left\{u_{k}\right\}_{k=1}^{\infty}$ denote the associated eigenfunctions which are assumed to form an orthonormal basis in $L^{2}(\Omega)$.

The convergence of the spectrum of (2) towards that of (3) has been proved in Gómez et al [1]. This result in Gómez et al [1] does not provide bounds for convergence rates of eigenvalues and the associated eigenfunctions, since it is obtained from general convergence results for nonlinear stationary problems, and convergence rates for the solutions of these stationary problems rely on the assumption of smoothness of the solution of the limiting problem. Since we are dealing with eigenvalue problems, such an assumption makes no sense.

The aim of this paper is to obtain precise bounds for discrepancies of the eigenvalues of (1) and (3) and for the associated eigenfunctions in terms of the eigenvalue number and the parameter $\varepsilon$. We emphasize that obtaining these bounds proves to be essential in order to determine, e.g., estimates of time in terms of $\varepsilon$. That is, the time in which certain solutions of the associated evolution problems can be approached through time-dependent functions constructed from (3) (see Pérez [5] and Pérez [6] in this connection). Associated evolution problems arise, e.g., in Ecology: see Gómez et al [1] for further references on the model and related works in the literature. For the proofs, we use a strong result from the spectral perturbation theory (cf. Lemma 2) for $\varepsilon$-dependent Hilbert spaces and operators, which provides convergence for the spectrum when a certain convergence for associated stationary problems is known.

In this paper, since we are dealing with a linear problem, we can obtain the required smoothness for the solution of the stationary problem (8) (cf. Lemma 3). Consequently, avoiding the assumptions on smoothness of solutions in Gómez et al [1] we obtain lower order powers of $\varepsilon$ in the bounds for the discrepancies but in these bounds we can control the dependence on the data $f$ in 
the norm of $L^{2}(\Omega)$ which is a usual topology for the spectral problems here considered (cf. (13) and (14)). To prove the above mentioned smoothness, we use a variant of results on interior estimates of Sobolev norms for solutions of second order elliptic equations with Dirichlet boundary conditions (cf. Shaposhnikov [7]), and Sobolev embedding theorems which also imply some restriction on the dimension of the space under consideration.

It should be noted that the critical case here considered where $k=\alpha=2$ provides the most singular limiting problem among all the possible relations between the parameters $\alpha$ and $\kappa$ in Gómez et al [1]: it appears a nonlinear dependence on the data $a(x)$ on the transmission condition on $\gamma$ (see (3)). Also, it should be emphasized that the technique here developed extends, for a three dimensional domain, to all the cases where $\alpha \geq 1$ and $\kappa \in \mathbb{R}$. The cases different from $\kappa=\alpha=2$ are considered in Gómez et al [2]. In these problems, different test functions are used and different homogenized spectral problems are obtained: either the average on $\gamma$ depends linearly on $a(x)$ or it does not contain any dependence on $a(x)$.

We note that the spectral problem here considered differs from others in the literature: in this respect, we refer to Gómez et al [2] for comparison. Finally, we mention that Section 2 contains some notations and preliminary results used to prove the convergence in Section 3. Theorem 4 contains the convergence results for the stationary problems and Theorem 5 contains the spectral convergence.

\section{Preliminary Results}

For the sake of the completeness, we introduce some lemmas bellow; in these lemmas, and in what follows, $C$ and $C_{k}$ denote constants independent of $\varepsilon$. Lemma 1 provides sharp general estimates for thin domains; Lemma 2 provides results from the spectral perturbation theory. Lemma 3 provides bounds in $W^{1,4}$ and $L^{\infty}$ norms for solutions.

Lemma 1. Let $\Pi_{\varepsilon}$ be $\Pi_{\varepsilon}=\Omega \cap\left\{-\varepsilon / 2<x_{1}<\varepsilon / 2\right\}$. Then, for all $w \in H_{0}^{1}(\Omega)$, we have

$$
\|w\|_{L^{2}\left(\Pi_{\varepsilon}\right)} \leq C \varepsilon^{1 / 2}\|\nabla w\|_{L^{2}(\Omega)} \quad \text { and } \quad\|w\|_{L^{4}\left(\Pi_{\varepsilon}\right)} \leq C \varepsilon^{1 / 8}\|\nabla w\|_{L^{2}(\Omega)} .
$$

Sketch of the proof. See, e.g., Lemma 2.6 in Gómez et al [1] for the first inequality in (4). The second one can be obtained from the Hölder inequality $\|w\|_{L^{4}\left(\Pi_{\varepsilon}\right)} \leq\|w\|_{L^{2}\left(\Pi_{\varepsilon}\right)}^{1 / 4}\|w\|_{L^{6}\left(\Pi_{\varepsilon}\right)}^{3 / 4}$, the first inequality in (4), and the embedding of $H_{0}^{1}(\Omega)$ into $L^{6}(\Omega)$. We refer to Gómez et al [2] for further explanation. 
Lemma 2. Let $H_{\varepsilon}$ and $H_{0}$ be two separable Hilbert spaces with the scalar products $(\cdot, \cdot)_{\varepsilon}$ and $(\cdot, \cdot)_{0}$ respectively. Let $A^{\varepsilon} \in \mathcal{L}\left(H_{\varepsilon}\right)$ and $A^{0} \in \mathcal{L}\left(H_{0}\right)$. Let $\mathcal{W}$ be a subspace of $H_{0}$ such that $\operatorname{Im} A^{0}=\left\{v \mid v=A^{0} u: u \in H_{0}\right\} \subset \mathcal{W}$. We assume that the following properties are satisfied:

(C1) There exists an operator $\mathcal{R}^{\varepsilon} \in \mathcal{L}\left(H_{0}, H_{\varepsilon}\right)$ such that, for any $f \in \mathcal{W}$, $\left\|\mathcal{R}^{\varepsilon} f\right\|_{\varepsilon} \rightarrow\|f\|_{0}$ as $\varepsilon \rightarrow 0$.

(C2) $A^{\varepsilon}$ and $A^{0}$ are positive, compact and self-adjoint operators on $H_{\varepsilon}$ and $H_{0}$ respectively. Besides, the norms $\left\|A^{\varepsilon}\right\|_{\mathcal{L}\left(H_{\varepsilon}\right)}$ are bounded by a constant independent of $\varepsilon$.

(C3) For any $f \in \mathcal{W},\left\|A^{\varepsilon} \mathcal{R}^{\varepsilon} f-\mathcal{R}^{\varepsilon} A^{0} f\right\|_{\varepsilon} \rightarrow 0$ as $\varepsilon \rightarrow 0$.

(C4) The family of operators $A^{\varepsilon}$ is uniformly compact, i.e., for any sequence $f^{\varepsilon}$ in $H_{\varepsilon}$ such that $\sup _{\varepsilon}\left\|f^{\varepsilon}\right\|_{\varepsilon}$ is bounded by a constant independent of $\varepsilon$, we can extract a subsequence $f^{\varepsilon^{\prime}}$ verifying $\left\|A^{\varepsilon^{\prime}} f^{\varepsilon^{\prime}}-\mathcal{R}^{\varepsilon^{\prime}} w^{0}\right\|_{\varepsilon^{\prime}} \rightarrow 0$, as $\varepsilon^{\prime} \rightarrow 0$, for certain $w^{0} \in \mathcal{W}$.

Let $\left\{\mu_{i}^{\varepsilon}\right\}_{i=1}^{\infty}\left(\left\{\mu_{i}^{0}\right\}_{i=1}^{\infty}\right.$, respectively) be the sequence of the eigenvalues of $A^{\varepsilon}\left(A^{0}\right.$, respectively) with the usual convention of repeated eigenvalues. Let $\left\{w_{i}^{\varepsilon}\right\}_{i=1}^{\infty}$ and $\left(\left\{w_{i}^{0}\right\}_{i=1}^{\infty}\right.$, respectively) be the corresponding eigenfunctions which are assumed to be an orthonormal basis in $H_{\varepsilon}$ ( $H_{0}$, respectively). Then, for each fixed $k$ there exist a constant $C_{k}$ and a $\varepsilon_{k}>0$ such that, for $\varepsilon \leq \varepsilon_{k}$,

$$
\left|\mu_{k}^{\varepsilon}-\mu_{k}^{0}\right| \leq C_{k} \sup \left\|A^{\varepsilon} \mathcal{R}^{\varepsilon} u-\mathcal{R}^{\varepsilon} A^{0} u\right\|_{\varepsilon},
$$

where the sup is taken over all the functions $u$ in the eigenspace associated with $\mu_{k}^{0}$, u such that $\|u\|_{0}=1$.

In addition, for any eigenvalue $\mu_{k}^{0}$ of $A^{0}$ with multiplicity $s\left(\mu_{k}^{0}=\mu_{k+1}^{0}=\right.$ $\left.\cdots=\mu_{k+s-1}^{0}\right)$, and for any $w$ eigenfunction corresponding to $\mu_{k}^{0}$, with $\|w\|_{0}=1$, there exists $w^{\varepsilon}, w^{\varepsilon}$ being a linear combination of eigenfunctions $\left\{w_{j}^{\varepsilon}\right\}_{j=k}^{j=k+s-1}$ of $A^{\varepsilon}$ corresponding to $\left\{\mu_{j}^{\varepsilon}\right\}_{j=k}^{j=k+s-1}$, such that

$$
\left\|w^{\varepsilon}-\mathcal{R}^{\varepsilon} w\right\|_{\varepsilon} \leq C_{k}\left\|A^{\varepsilon} \mathcal{R}^{\varepsilon} w-\mathcal{R}^{\varepsilon} A^{0} w\right\|_{\varepsilon} .
$$

We refer to Theorems 1.4 and 1.7 in Chapter III of Oleinik [4] for the proof of Lemma 2.

Let us define $\mathcal{H}^{\varepsilon}=L^{2}\left(\Omega_{\varepsilon}\right)$ and $\mathcal{H}^{0}=L^{2}(\Omega)$ with the usual scalar products. Let us introduce the operators $\mathcal{A}^{\varepsilon}: \mathcal{H}^{\varepsilon} \rightarrow \mathcal{H}^{\varepsilon}$ and $\mathcal{A}^{0}: \mathcal{H}^{0} \rightarrow \mathcal{H}^{0}$. For $f^{\varepsilon} \in \mathcal{H}^{\varepsilon}$, 
we set $\mathcal{A}^{\varepsilon} f^{\varepsilon}=u_{\varepsilon}$ where $u_{\varepsilon} \in H^{1}\left(\Omega_{\varepsilon}, \partial \Omega\right)$ is the unique solution of

$$
\int_{\Omega_{\varepsilon}} \nabla u_{\varepsilon} \nabla v d x+\varepsilon^{-\kappa} \int_{S_{\varepsilon}} a u_{\varepsilon} v d s=\int_{\Omega_{\varepsilon}} f^{\varepsilon} v d x, \quad \forall v \in H^{1}\left(\Omega_{\varepsilon}, \partial \Omega\right) .
$$

Consequently, the eigenelements of $\mathcal{A}^{\varepsilon}$ are $\left\{\left(\left(\lambda_{k}^{\varepsilon}\right)^{-1}, u_{k}^{\varepsilon}\right)\right\}_{k=1}^{\infty}$ with $\left\{\left(\lambda_{k}^{\varepsilon}, u_{k}^{\varepsilon}\right)\right\}_{k=1}^{\infty}$ the eigenelements of (2). In the same way, for $f \in \mathcal{H}^{0}$, we set $\mathcal{A}^{0} f=u$ where $u \in H_{0}^{1}(\Omega)$ is the unique solution of

$$
\int_{\Omega} \nabla u \nabla v d x+4 \pi C_{0} \int_{\gamma} h u v d \hat{x}=\int_{\Omega} f v d x, \quad \forall v \in H_{0}^{1}(\Omega),
$$

and, the eigenelements of $\mathcal{A}^{0}$ are $\left\{\left(\left(\lambda_{k}\right)^{-1}, u_{k}\right)\right\}_{k=1}^{\infty}$ with $\left\{\left(\lambda_{k}, u_{k}\right)\right\}_{k=1}^{\infty}$ the eigenelements of (3). Above $\hat{x}$ denotes $\hat{x}=\left(x_{2}, x_{3}\right)$. We also set $\mathcal{W}=H_{0}^{1}(\Omega)$.

Finally, we define $\mathcal{R}^{\varepsilon}: L^{2}(\Omega) \rightarrow L^{2}\left(\Omega_{\varepsilon}\right)$ the restriction operator; namely, $\left(\mathcal{R}^{\varepsilon} f\right)(x)=f(x)$ if $x \in \Omega_{\varepsilon}$.

Let us introduce the extension operator $\mathcal{P}_{\varepsilon}$ from $H^{1}\left(\Omega_{\varepsilon}, \partial \Omega\right)$ into $H_{0}^{1}(\Omega)$, such that for $w \in H^{1}\left(\Omega_{\varepsilon}, \partial \Omega\right)$ we set $\mathcal{P}_{\varepsilon} w=\widetilde{w}$ the function which satisfies: $\widetilde{w}(x)=w(x)$ for $x \in \Omega_{\varepsilon}$, and

$$
\|\widetilde{w}\|_{H^{1}(\Omega)} \leq C\|w\|_{H^{1}\left(\Omega_{\varepsilon}\right)} \quad \text { and } \quad\|\nabla \widetilde{w}\|_{L^{2}(\Omega)} \leq C\|\nabla w\|_{L^{2}\left(\Omega_{\varepsilon}\right)} .
$$

This allows us to prove the estimates for $u_{\varepsilon}=\mathcal{A}^{\varepsilon}\left(\mathcal{R}^{\varepsilon} f\right.$ ) (see Lemma 2.7 and Theorem 2.1 in Gómez et al [1]):

$$
\begin{aligned}
& \left\|\nabla u_{\varepsilon}\right\|_{L^{2}\left(\Omega_{\varepsilon}\right)}+\varepsilon^{-\kappa / 2}\left\|u_{\varepsilon}\right\|_{L^{2}\left(S_{\varepsilon}\right)} \leq C\|f\|_{L^{2}\left(\Omega_{\varepsilon}\right)}, \\
& \left\|\widetilde{u}_{\varepsilon}\right\|_{H^{1}(\Omega)} \leq C\|f\|_{L^{2}\left(\Omega_{\varepsilon}\right)} .
\end{aligned}
$$

Lemma 3. Let $u$ be the solution of (8) with $f \in L^{2}(\Omega)$. Then,

$$
\begin{aligned}
& \|u\|_{H^{1}(\Omega)} \leq C\|f\|_{L^{2}(\Omega)}, \\
& \|u\|_{W^{1,4}(\Omega)} \leq C\|f\|_{L^{2}(\Omega)}, \\
& \|u\|_{L^{\infty}(\Omega)} \leq C\|f\|_{L^{2}(\Omega)} .
\end{aligned}
$$

Sketch of the proof. From the Poincaré inequality, taking $v=u$ in (8), we get the first estimate in (11). For the second estimate, we consider the function $\psi(x)=u(x) \exp (g(x))$ where $u$ is the solution of (8) and $g$ is defined by $g(x)=-4 \pi C_{0} h(0, \hat{x}) x_{1}$ if $x_{1}>0$ and $g(x)=0$ otherwise. Then, we show 
that $\psi$ satisfies the equation $-\partial_{x_{i}}\left(c \partial_{x_{i}} \psi+b_{i} \psi\right)=f$ in $\Omega$, for certain functions $c \in C^{0,1}(\Omega)$ and $b_{i} \in L^{\infty}(\Omega), i=1,2,3$, and the Dirichlet condition $\psi=0$ on $\partial \Omega$. Then, using an adaptation of the proof in Theorem 1 in Shaposhnikov [7] for $n=3, q=4$ and $p=12 / 7$ (see also Section V.5 in Morrey [3]) we have $\|\psi\|_{W^{1,4}(\Omega)} \leq C\left(\|\psi\|_{L^{1}(\Omega)}+\|f\|_{L^{12 / 7}(\Omega)}\right)$. We refer to Gómez et al [2] for further explanation.

Consequently, by definition of $\psi$, the smoothness of $h$, the embedding of the spaces $L^{r}$ with $1 \leq r \leq \infty$, and the first estimate in (11), we obtain the second estimate in (11). The last estimate in (11) can be obtained directly from the second estimate, namely the estimate for the $W^{1,4}(\Omega)$-norm, and the embedding of $W^{1,4}(\Omega)$ into $L^{\infty}(\Omega)$.

Finally, in order to prove the convergence, we introduce the test function $W_{\varepsilon}$. Let $P_{\varepsilon}^{j}$ be the center of the ball $G_{\varepsilon}^{j}$ and we denote by $T_{\varepsilon}^{j}$ the ball of radius $\varepsilon / 4$ with center $P_{\varepsilon}^{j}$. Let us consider the functions $w_{\varepsilon}^{j}\left(j \in \Upsilon_{\varepsilon}\right)$ as the solutions of the following problems

$$
\Delta w_{\varepsilon}^{j}=0 \text { in } T_{\varepsilon}^{j} \backslash \overline{G_{\varepsilon}^{j}}, \quad w_{\varepsilon}^{j}=1 \text { on } \partial G_{\varepsilon}^{j}, \quad w_{\varepsilon}^{j}=0 \text { on } \partial T_{\varepsilon}^{j} .
$$

We define the function $W_{\varepsilon} \in H^{1}\left(\mathbb{R}^{n}\right)$ by extending by 1 for $x \in \overline{G_{\varepsilon}}$ and by 0 for $x \in \mathbb{R}^{n} \backslash \bigcup_{j \in \Upsilon_{\varepsilon}} T_{\varepsilon}^{j}$. As is well-known (see, e.g., Gómez et al [1]), the solution of (12) can be constructed explicitly, $0 \leq W_{\varepsilon} \leq 1$, and the weak convergence $W_{\varepsilon} \rightarrow 0$ in $H_{0}^{1}(\Omega)$, as $\varepsilon \rightarrow 0$, holds.

\section{Convergence Results when $\alpha=\kappa=2$}

Theorem 4. Let $W_{\varepsilon}$ and $h$ be the functions defined by (12) and (3) respectively. For $f \in L^{2}(\Omega)$, let $u$ be the solution of (8) and let $u_{\varepsilon}$ be the solution of (7) for $f^{\varepsilon}$ equal to the restriction of $f$ to $\Omega_{\varepsilon}$. Then, we have

$$
\left\|u_{\varepsilon}-u+W_{\varepsilon} h u\right\|_{H^{1}\left(\Omega_{\varepsilon}\right)}^{2}+\varepsilon^{-2}\left\|u_{\varepsilon}-u+h u\right\|_{L^{2}\left(S_{\varepsilon}\right)}^{2} \leq C \varepsilon^{1 / 8}\|f\|_{L^{2}(\Omega)}^{2}
$$

and

$$
\left\|u_{\varepsilon}-u\right\|_{L^{2}\left(\Omega_{\varepsilon}\right)}^{2} \leq C \varepsilon^{1 / 8}\|f\|_{L^{2}(\Omega)}^{2}
$$

Proof. Let us consider (7) and (8) with $v=u_{\varepsilon}-u+W_{\varepsilon} h u \in H^{1}\left(\Omega_{\varepsilon}, \partial \Omega\right)$ and $v=\widetilde{u}_{\varepsilon}-u+W_{\varepsilon} h u \in H_{0}^{1}(\Omega)$ as test functions, respectively. Subtracting both equalities, we obtain

$$
\left\|\nabla\left(u_{\varepsilon}-u+W_{\varepsilon} h u\right)\right\|_{L^{2}\left(\Omega_{\varepsilon}\right)}^{2}+\varepsilon^{-2} \int_{S_{\varepsilon}} a\left(u_{\varepsilon}-u+h u\right)^{2} d s=S_{1}+S_{2}+S_{3},
$$


where

$$
\begin{aligned}
S_{1}=\int_{G_{\varepsilon}} \nabla u \nabla\left(\widetilde{u}_{\varepsilon}-u\right. & \left.+W_{\varepsilon} h u\right) d x, \quad S_{2}=-\int_{G_{\varepsilon}} f\left(\widetilde{u}_{\varepsilon}-u+W_{\varepsilon} h u\right) d x \\
S_{3}= & \int_{\Omega_{\varepsilon}} \nabla\left(W_{\varepsilon} h u\right) \nabla\left(u_{\varepsilon}-u+W_{\varepsilon} h u\right) d x \\
& +4 \pi C_{0} \int_{\gamma} h u\left(\widetilde{u}_{\varepsilon}-u+W_{\varepsilon} h u\right) d \hat{x} \\
& -\varepsilon^{-2} \int_{S_{\varepsilon}} a(1-h) u\left(u_{\varepsilon}-u+h u\right) d s .
\end{aligned}
$$

Now, considering the volume of $G_{\varepsilon}$, the definition and the boundedness of $W_{\varepsilon}$ in $H^{1}(\Omega)$, the smoothness of $h,(11),(10)$ and (4), we obtain

$$
\begin{gathered}
\left|S_{1}\right| \leq\|\nabla u\|_{L^{4}\left(G_{\varepsilon}\right)}\left|G_{\varepsilon}\right|^{1 / 4}\left\|\nabla\left(\widetilde{u}_{\varepsilon}-u+W_{\varepsilon} h u\right)\right\|_{L^{2}(\Omega)} \leq C \varepsilon\|f\|_{L^{2}(\Omega)}^{2} \text { and } \\
\left|S_{2}\right| \leq\|f\|_{L^{2}\left(G_{\varepsilon}\right)} \varepsilon^{1 / 2}\left\|\nabla\left(\widetilde{u}_{\varepsilon}-u+W_{\varepsilon} h u\right)\right\|_{L^{2}(\Omega)} \leq C \varepsilon^{1 / 2}\|f\|_{L^{2}(\Omega)}^{2} .
\end{gathered}
$$

Let us estimate $S_{3}$. Using

$$
\begin{aligned}
\int_{\Omega_{\varepsilon}} \nabla\left(W_{\varepsilon} h u\right) \nabla w d x= & \int_{\Omega_{\varepsilon}} \nabla W_{\varepsilon} \nabla(h u w) d x-\int_{\Omega_{\varepsilon}} \nabla W_{\varepsilon} \nabla(h u) w d x \\
& +\int_{\Omega_{\varepsilon}} W_{\varepsilon} \nabla(h u) \nabla w d x
\end{aligned}
$$

for $w=u_{\varepsilon}-u+W_{\varepsilon} h u$, the Green formula in the first integral on the right hand side above, and the definition of $W_{\varepsilon}$ we have that $S_{3}=S_{3 a}+S_{3 b}+S_{3 c}$ where

$$
\begin{aligned}
S_{3 a}= & \sum_{j \in \Upsilon_{\varepsilon}} \int_{\partial T_{\varepsilon}^{j}} \partial_{\nu} w_{\varepsilon}^{j} h u\left(u_{\varepsilon}-u+W_{\varepsilon} h u\right) d s \\
& +4 \pi C_{0} \int_{\gamma} h u\left(\widetilde{u}_{\varepsilon}-u+W_{\varepsilon} h u\right) d \hat{x},
\end{aligned}
$$




$$
\begin{aligned}
S_{3 b}= & \sum_{j \in \Upsilon_{\varepsilon}} \int_{\partial G_{\varepsilon}^{j}} \partial_{\nu} w_{\varepsilon}^{j} h u\left(u_{\varepsilon}-u+W_{\varepsilon} h u\right) d s \\
& -\varepsilon^{-2} \int_{S_{\varepsilon}} a(1-h) u\left(u_{\varepsilon}-u+h u\right) d s, \\
S_{3 c}= & -\int_{\Omega_{\varepsilon}} \nabla W_{\varepsilon} \nabla(h u)\left(u_{\varepsilon}-u+W_{\varepsilon} h u\right) d x \\
& +\int_{\Omega_{\varepsilon}} W_{\varepsilon} \nabla(h u) \nabla\left(u_{\varepsilon}-u+W_{\varepsilon} h u\right) d x .
\end{aligned}
$$

Taking into account the explicit computation of the normal derivatives of $w_{\varepsilon}^{j}$, the estimate

$$
\left|\sum_{j \in \Upsilon_{\varepsilon}} \int_{\partial T_{\varepsilon}^{j}} w d s-\frac{\pi}{4} \int_{\gamma} w d \hat{x}\right| \leq C \varepsilon^{1 / 2}\|\nabla w\|_{L^{2}(\Omega)}, \quad \forall w \in H_{0}^{1}(\Omega)
$$

(see Lemma 2.5 in Gómez et al [1] for the proof), and the trace theorem in $H^{1}(\Omega)$, we have

$$
\left|S_{3 a}\right| \leq C \varepsilon^{1 / 2}\left\|\nabla\left(h u\left(\widetilde{u}_{\varepsilon}-u+W_{\varepsilon} h u\right)\right)\right\|_{L^{2}(\Omega)} .
$$

In addition, from the smoothness of $h$, the embedding theorem of $H_{0}^{1}(\Omega)$ into $L^{6}(\Omega)$, the boundedness of $W_{\varepsilon}$ in $H^{1}(\Omega),(10)$ and (11), it follows that

$$
\begin{aligned}
\left|S_{3 a}\right| \leq & C \varepsilon^{1 / 2}\left(\|\nabla u\|_{L^{4}(\Omega)}\left\|\widetilde{u}_{\varepsilon}-u+W_{\varepsilon} h u\right\|_{L^{4}(\Omega)}\right. \\
& \left.+\|u\|_{L^{\infty}(\Omega)}\left\|\nabla\left(\widetilde{u}_{\varepsilon}-u+W_{\varepsilon} h u\right)\right\|_{L^{2}(\Omega)}\right) \leq C \varepsilon^{1 / 2}\|f\|_{L^{2}(\Omega)}^{2} .
\end{aligned}
$$

Finally, by the definition of $W_{\varepsilon}$ and $h$, we can rewrite $S_{3 b}$ as

$$
S_{3 b}=\frac{4}{\varepsilon-4 C_{0} \varepsilon^{2}} \int_{S_{\varepsilon}} h u\left(u_{\varepsilon}-u+h u\right) d s .
$$

Thus, computing the area of $S_{\varepsilon}$ and using (11) and (10) we get

$$
\begin{aligned}
\left|S_{3 b}\right| & \leq C \varepsilon^{-1}\left(\|u\|_{L^{\infty}(\Omega)}\left|S_{\varepsilon}\right|^{1 / 2}\left\|u_{\varepsilon}\right\|_{L^{2}\left(S_{\varepsilon}\right)}+\|u\|_{L^{\infty}(\Omega)}^{2}\left|S_{\varepsilon}\right|\right) \\
& \leq C \varepsilon\|f\|_{L^{2}(\Omega)}^{2} .
\end{aligned}
$$


In a similar way,

$$
\begin{aligned}
\left|S_{3 c}\right| \leq & \left\|\nabla W_{\varepsilon}\right\|_{L^{2}(\Omega)}\|\nabla(h u)\|_{L^{4}\left(\Pi_{\varepsilon}\right)}\left\|\widetilde{u}_{\varepsilon}-u+W_{\varepsilon} h u\right\|_{L^{4}\left(\Pi_{\varepsilon}\right)} \\
& +\left|\Pi_{\varepsilon}\right|^{1 / 4}\|\nabla(h u)\|_{L^{4}\left(\Pi_{\varepsilon}\right)}\left\|\nabla\left(\widetilde{u}_{\varepsilon}-u+W_{\varepsilon} h u\right)\right\|_{L^{2}\left(\Pi_{\varepsilon}\right)},
\end{aligned}
$$

and by the boundedness of $W_{\varepsilon}$ in $H^{1}(\Omega),(11),(10)$ and (4) we get

$$
\left|S_{3 c}\right| \leq C \varepsilon^{1 / 8}\|f\|_{L^{2}(\Omega)}^{2} .
$$

Now, gathering all the above estimates, we conclude that

$$
\begin{gathered}
\left\|\nabla\left(u_{\varepsilon}-u+W_{\varepsilon} h u\right)\right\|_{L^{2}\left(\Omega_{\varepsilon}\right)}^{2}+\varepsilon^{-2}\left\|u_{\varepsilon}-u+h u\right\|_{L^{2}\left(S_{\varepsilon}\right)}^{2} \\
\leq C \varepsilon^{1 / 8}\|f\|_{L^{2}(\Omega)}^{2} .
\end{gathered}
$$

To obtain (13) from (15), we consider the Poincaré inequality for the $H^{1}$ extension of $u_{\varepsilon}-u+W_{\varepsilon} h u$ to $\Omega, \mathcal{P}_{\varepsilon}\left(u_{\varepsilon}-u+W_{\varepsilon} h u\right) \in H_{0}^{1}(\Omega)$, which satisfies (9) for $w=u_{\varepsilon}-u+W_{\varepsilon} h u$.

Finally, from (13), the definition of $W_{\varepsilon}$, the smoothness of $h,(4)$ and (11), we can write

$$
\begin{aligned}
\left\|u_{\varepsilon}-u\right\|_{L^{2}\left(\Omega_{\varepsilon}\right)}^{2} & \leq\left\|u_{\varepsilon}-u+W_{\varepsilon} h u\right\|_{L^{2}\left(\Omega_{\varepsilon}\right)}^{2}+\left\|W_{\varepsilon} h u\right\|_{L^{2}\left(\Omega_{\varepsilon}\right)}^{2} \\
& \leq C\left(\varepsilon^{1 / 8}\|f\|_{L^{2}(\Omega)}^{2}+\|u\|_{L^{2}\left(\Pi_{\varepsilon}\right)}^{2}\right) \\
& \leq C\left(\varepsilon^{1 / 8}\|f\|_{L^{2}(\Omega)}^{2}+\varepsilon\|\nabla u\|_{L^{2}(\Omega)}^{2}\right) \leq C \varepsilon^{1 / 8}\|f\|_{L^{2}(\Omega)}^{2} .
\end{aligned}
$$

Consequently, (14) holds and the theorem is proved.

Theorem 5. Let $\left\{\lambda_{k}^{\varepsilon}\right\}_{k=1}^{\infty}$ and $\left\{\lambda_{k}\right\}_{k=1}^{\infty}$ be the eigenvalues of problem (1) and (3), respectively. Then, for each fixed $k$ there exists a constant $C_{k}$ independent of $\varepsilon$ such that

$$
\left|\lambda_{k}^{\varepsilon}-\lambda_{k}\right| \leq C_{k} \varepsilon^{1 / 16}
$$

holds for sufficiently small $\varepsilon$. In addition, for any eigenvalue $\lambda_{k}$ of (3) with multiplicity $s\left(\lambda_{k}=\lambda_{k+1}=\cdots=\lambda_{k+s-1}\right)$, and for any $u$ eigenfunction corresponding to $\lambda_{k}$, with $\|u\|_{L^{2}(\Omega)}=1$, there exists $\tilde{u}^{\varepsilon}, \tilde{u}^{\varepsilon}$ a linear combination of eigenfunctions $\left\{u_{k}^{\varepsilon}\right\}_{r=k}^{r=k+s-1}$ of (1) corresponding to $\left\{\lambda_{k}^{\varepsilon}\right\}_{r=k}^{r=k+s-1}$, such that

$$
\left\|\tilde{u}^{\varepsilon}-u\right\|_{L^{2}\left(\Omega_{\varepsilon}\right)} \leq C_{k} \varepsilon^{1 / 16}
$$


Proof. On account of (10) and (14), it is self-evident that the properties $(C 1)-(C 3)$ of Lemma 2 are satisfied. Let us prove property $(C 4)$ in Lemma 2. In order to do this, for the $f^{\varepsilon} \in L^{2}\left(\Omega_{\varepsilon}\right)$, as stated in property $(C 4)$, we consider $\widehat{f^{\varepsilon}} \in L^{2}(\Omega)$ the extension of $f^{\varepsilon}$ by zero inside $G_{\varepsilon}$. We have that $\left\|\widehat{f}^{\varepsilon}\right\|_{L^{2}(\Omega)}$ is bounded by a constant independent of $\varepsilon$ and consequently, there is a subsequence $\varepsilon^{\prime} \rightarrow 0$ and a certain $f^{0} \in L^{2}(\Omega)$ such that $\widehat{f}^{\varepsilon^{\prime}} \rightarrow f^{0}$ in $L^{2}(\Omega)$. Considering $u_{\varepsilon^{\prime}}=\mathcal{A}^{\varepsilon^{\prime}} \mathcal{R}^{\varepsilon^{\prime}} \widehat{f}^{\varepsilon^{\prime}}$ and $w^{0} \in H_{0}^{1}(\Omega)$ solution of (8) for $f=f^{0}$, we rewrite the proof in Theorem 4 with minor modifications, and we obtain that $\left\|u_{\varepsilon^{\prime}}-w^{0}\right\|_{L^{2}\left(\Omega_{\varepsilon}\right)} \rightarrow 0$, as $\varepsilon^{\prime} \rightarrow 0$. Consequently, property (C4) also holds.

Now, applying Lemma 2, we have that for each fixed $k$,

$$
\left|\left(\lambda_{k}^{\varepsilon}\right)^{-1}-\left(\lambda_{k}\right)^{-1}\right| \leq C_{k} \sup \left\|u_{\varepsilon, k}-u_{0, k}\right\|_{L^{2}\left(\Omega_{\varepsilon}\right)}
$$

where the sup is taken over all the functions $f_{k}$ in the eigenspace associated with $\left(\lambda_{k}\right)^{-1}, f_{k}$ such that $\left\|f_{k}\right\|_{L^{2}(\Omega)}=1, u_{\varepsilon, k}$ and $u_{0, k}$ are $u_{\varepsilon, k}=\mathcal{A}^{\varepsilon} \mathcal{R}^{\varepsilon} f_{k}$ and $u_{0, k}=\mathcal{R}^{\varepsilon} \mathcal{A}^{0} f_{k}$ respectively. But, (14) allows us to assert

$$
\left\|u_{\varepsilon, k}-u_{0, k}\right\|_{L^{2}\left(\Omega_{\varepsilon}\right)}^{2} \leq C_{k} \varepsilon^{1 / 8}\left\|f_{k}\right\|_{L^{2}(\Omega)}^{2} \leq C_{k} \varepsilon^{1 / 8}
$$

for $C_{k}$ a certain constant independent of $\varepsilon$. From this last inequality, (18) reads $\left|\left(\lambda_{k}^{\varepsilon}\right)^{-1}-\left(\lambda_{k}\right)^{-1}\right|^{2} \leq C_{k} \varepsilon^{1 / 8}$ which ensures the boundedness of $\left(\lambda_{k}^{\varepsilon}\right)^{-1}$ by a constant independent of $\varepsilon$ and consequently the estimate for the eigenvalues (16) holds.

Finally, let us note that the estimate for the eigenfunctions (17) also holds applying (6) and (14).

\section{Acknowledgements}

This work has been partially supported by the Spanish project MTM200912628. The authors are grateful to Professor S.V. Shaposhnikov for our fruitful discussions.

\section{References}

[1] D. Gómez, E. Pérez, T.A. Shaposhnikova, On homogenization of nonlinear Robin type boundary conditions for cavities along manifolds and associated spectral problems, Asymptot. Anal., 80 (2012), 289-322. 
[2] D. Gómez, E. Pérez, T.A. Shaposhnikova, Spectral boundary homogenization problems in perforated domains with Robin boundary conditions and large parameters, In: Integral Methods in Science and Engineering, Birkhäuser (2013), 20 pp. (in press).

[3] C.B. Morrey, Multiple Integrals in the Calculus of Variations, SpringerVerlag, New York (1966).

[4] O.A. Oleinik, A.S. Shamaev, G.A. Yosifian, Mathematical Problems in Elasticity and Homogenization, North-Holland, Amsterdam (1992).

[5] E. Pérez, Long time approximations for solutions of wave equations via standing waves from quasimodes, J. Math. Pures Appl., 90 (2008), 387411.

[6] E. Pérez, Long time approximations for solutions of evolution equations from quasimodes: Perturbation problems, Math. Balkanica, 25 (2011), $95-130$.

[7] S.V. Shaposhnikov, On interior estimates of the Sobolev norms of solutions of elliptic equations, Math. Notes, 83 (2008), 285-289. 